PHYSICAL REVIEW D 94, 029903(E) (2016)

\title{
Erratum: Diffeomorphism-invariant observables and their nonlocal algebra [Phys. Rev. D 93, 024030 (2016)]
}

William Donnelly and Steven B. Giddings

(Received 15 July 2016; published 27 July 2016)

DOI: 10.1103/PhysRevD.94.029903

Equation (69) incorrectly used the canonical momentum $\pi^{i j}$ derived from the gauge-fixed action in Feynman gauge, rather than the canonical momentum derived from the ADM action. To linear order about flat spacetime, the latter is given by

$$
\pi_{\mathrm{ADM}}^{i j}=\dot{h}_{i j}-\partial_{i} h_{0 j}-\partial_{j} h_{0 i}-\dot{h}_{k k} \delta_{i j}+2 \partial_{k} h_{0 k} \delta_{i j} .
$$

The corrected equation (69) should then read

$$
P_{\mathrm{ADM}}^{i}=-\frac{2}{\kappa} \oint r^{2} d^{2} \Omega \hat{r}_{j} \pi_{\mathrm{ADM}}^{i j}=-\frac{2}{\kappa} \oint r^{2} d^{2} \Omega \hat{r}^{j}\left(\dot{h}_{i j}+\partial_{i} h_{0 j}-\partial_{j} h_{0 i}-\dot{h}_{k k} \delta_{i j}\right),
$$

where we have used the identity $\oint r^{2} d^{2} \Omega \hat{r}^{j}\left(\partial_{k} h_{0 k} \delta_{i j}\right)=\oint r^{2} d^{2} \Omega \hat{r}^{j}\left(\partial_{i} h_{0 j}\right)$. The commutation relation (70) and the remainder of the paper are unaffected by this change. 